\title{
Oral contraceptives in the prevention of endometrioma recurrence: does the different progestins used make a difference?
}

\author{
Gaspare Cucinella $\cdot$ Roberta Granese $\cdot$ Gloria Calagna $\cdot$ Alessandro Svelato • \\ Salvatore Saitta $\cdot$ Gabriele Tonni $\cdot$ Pasquale De Franciscis · Nicola Colacurci • \\ Antonino Perino
}

Received: 14 January 2013/Accepted: 1 April 2013/Published online: 12 April 2013

(C) Springer-Verlag Berlin Heidelberg 2013

\begin{abstract}
Objective The primary aim of the study was to analyze the endometrioma recurrence rate in patients who underwent laparoscopic excision followed by postoperative longterm regimen of oral contraceptives (OCs).

Materials and methods 168 patients who underwent a conservative laparoscopic surgery for endometrioma, during the period between September 2009 and August 2010 in three university hospitals were studied. A long-term OCs therapy was offered to all women following surgery. Patients were randomly divided into three groups according to different progestins used (desogestrel, gestodene, dienogest). Women who refused a postoperative hormonal therapy served as control. Follow-up visits and transvaginal scan were planned at $1,3,6,12$, and 24 months after surgery. All patients who showed an ultrasound persistence of the endometrioma at 1 month follow-up were excluded from clinical analysis.
\end{abstract}

G. Cucinella and R. Granese are equal first authors.

G. Cucinella $\cdot$ G. Calagna $\cdot$ A. Svelato $\cdot$ A. Perino

Department of Obstetrics and Gynecology, University Hospital

"Paolo Giaccone", Palermo, Italy

R. Granese · S. Saitta · P. De Franciscis

Department of Obstetrics and Gynecology, University Hospital

"Gaetano Martino", Messina, Italy

G. Tonni $(\bowtie)$

Department of Obstetrics and Gynecology, Guastalla Civil

Hospital, AUSL Reggio Emilia, Via Donatori Sangue, 2,

42016 Guastalla, Reggio Emilia, Italy

e-mail: tonni.gabriele@ausl.re.it

N. Colacurci

Department of Obstetrics, Gynecology, and Reproductive

Sciences, Second University of Naples, Naples, Italy
Results Of the 168 patients, 131 completed the 24 months follow-up. Endometrioma recurrence was found in $21(12.5 \%)$ of all patients, it was unilateral in 17 cases while bilateral in 4 cases. The rate of recurrent endometrioma was statistically significant in non-users compared to the long-term OCs treated patients.

Conclusion The current data suggest the usefulness of long-term OCs regimen after conservative surgery for the prevention of ovarian endometrioma recurrence. As a statistical significant difference could not be observed between OCs groups, further study on the individual molecules is required in order to really understand the effect of each of them.

Keywords Endometriosis - Endometrioma recurrence · Oral contraceptives $\cdot$ Minimally invasive gynaecologic surgery $\cdot$ Progestins $\cdot$ Dienogest

\section{Introduction}

Endometriosis is an interesting, chronic disease caused by the growth of functional endometrial-like tissue outside the uterus, which in turn causes infertility and pelvic pain, and affects up to $10 \%$ of women of reproductive age [1]. Ovary is the most common organ involved (in $\sim 55 \%$ of cases [2]). "Unfortunately, there is no permanent cure for this condition, so it often requires a life-long management plan with the goal of maximizing the use of medical treatment and avoiding repeated surgical procedures", reports the American Society of Reproductive Medicine [3]. Lesions and symptoms very frequently return after medication is discontinued or after conservative surgery is performed, a fact which frustrates both patients and clinicians [4] and is estimated to occur in $30-50 \%$ of cases 
within 2-5 years [2, 5-10]. The American College of Obstetricians and Gynecologists Practice Bulletin suggests that the true recurrence rate must be even higher than the rate generally reported in literature, because most publications report symptomatic disease only [11]. Conservative laparoscopic surgery is considered to be the gold standard for the treatment of ovarian endometriosis [12-17]. There is general agreement that endometrioma removal increases the likelihood of conception [18, 19], and recent evidence shows that both the presence of an endometrioma and its surgical excision may impair ovarian reserve and, therefore, future fertility [20,21], especially after a second operation. Although surgery is cytoreductive, it does not prevent recurrence, so therapeutic strategies should be found based on factors which affect the natural course of the disease and its tendency to reappear [5].

Moreover, since long-term medical therapy is necessary, consideration has been given not only to efficacy, but also to the long-term safety and tolerability of the treatment options available. For these reasons, oral contraceptives (OCs) have been widely used in many countries to treat the most common symptoms of endometriosis [22].

Some studies have reported that monophasic oral contraceptive treatment either to reduce retrograde menstrual cycles or to inhibit ovulation-which are both considered etiopathogenetic factors for endometriosis-may also suppress the recurrence of endometrioma when administered long term after conservative surgery [2, 5, 10, 16, 23]. Progestogens have a crucial role in decreasing endometriotic lesions, through a number of mechanisms which may vary in type and intensity according to the different molecules. They may prevent implantation and growth of regurgitated endometrium inhibiting expression of matrix metalloproteinases and angiogenesis, and have several antiinflammatory in vitro and in vivo effects that may reduce the inflammatory state generated by the metabolic activity of the ectopic endometrium, and the consequent immune response [24]. For this reason, new progestins are continually tested, in an attempt to find the perfect drug which ensures the desired effects without side effects. Two recent clinical studies in Europe and Japan extensively investigated a new treatment for endometriosis using Dienogest, a 19-nortestosterone derivative that is highly selective for progesterone receptors [25]. The studies demonstrated that at $2 \mathrm{mg}$ daily, this progestin effectively alleviates pain, reduces endometriosis lesions and improves quality of life [26]. Our controlled randomized study based on these reports was intended to analyze the recurrence rate of endometrioma in patients who had undergone laparoscopic excision and a long-term postoperative regimen of OCs to verify their effectiveness in preventing the disease. We also sought to establish whether a different progestin in the oral contraceptive could control recurrence in a different manner. Thus, we administered three types of OCs, which included dienogest.

\section{Materials and methods}

This multi-centre study was conducted in the Departments of Obstetrics and Gynaecology at the Universities of Naples, Messina and Palermo, Italy. A total of 168 patients who had undergone conservative surgery for ovarian endometrioma between September 2009 and August 2010 were enrolled. Permission was obtained from the Local Institutional Review Board before the study began.

Women between 18 and 40 years, not attempting to conceive, either at the time of study entry or for at least 2 years after surgery, were included in the study. We excluded patients who had previously undergone surgical treatment for endometriosis or had taken recent medical treatment for this pathology. Therefore, none of them had been receiving OCs for at least 6 months before surgery. We also excluded patients with contraindications to OCs therapy or who did not wish to postpone pregnancy for at least 2 years after surgery.

After transvaginal ultrasound diagnosis, the women underwent laparoscopic excision of the endometrioma using the so-called "stripping technique". The procedures were performed by surgeons with expertise in minimally invasive gynaecologic surgery listed as Level II by the SEGI (Italian Society of Gynaecologic Endoscopy). The staging of the endometriosis was carried out using the procedure specified by the American Society for Reproductive Medicine (ASRM) [27].

Upon hospital discharge, long-term therapy (24 months) with OCs was offered to all patients, who were advised that inhibition of ovulation may potentially reduce the risk of recurrence, according to recent medical evidence. They were asked to participate in the study and signed an informed consent form. Treatment allocation was performed in accordance with a computer-generated randomization sequence with the use of numbered, opaque, sealed envelopes.

The primary aim of the study was to assess any statistical differences of clinical import among the types of progestins used. Two monophasic and one multiphasic OC were selected. Patients who did not accept the treatment protocol were considered to be "non-users" (Group A, 38 patients) and were scheduled to undergo the same followup protocol.

The remaining 130 patients were randomly divided into three subgroups according to progestin type: monophasic pill with EE $20 \mu \mathrm{g}$ and $0.15 \mathrm{mg}$ desogestrel daily (Group B, 43 patients); monophasic pill with EE $20 \mu \mathrm{g}$ and gestodene $0.075 \mathrm{mg}$ daily (Group C, 44 patients); and 
multiphasic pill with $2 \mathrm{mg}$ E2V for 22 days, with $2 \mathrm{mg}$ dienogest for the first 5 days and $3 \mathrm{mg}$ on the remaining 17 days, the other four: pill with only E2V and two: placebo pill (Group D, 43 patients). The patients were followed up with a clinical examination and a transvaginal ultrasonographic scan, for at least 24 months. Follow-up visits were planned at $1,3,6,12$, and 24 months after surgery. All the scans were performed by experienced operators, blinded to the study allocation.

The secondary aim of the study was to assess the timing of recurrence by calculating (in months) the reappearance of the ovarian endometrioma after surgery.

The presence of an endometrioma 1 month after surgery, as diagnosed by transvaginal ultrasound, was considered to be persistence of the disease, and these patients were excluded from follow-up. The diagnostic ultrasonographic criteria established by Mais et al. [28] for endometrioma recurrence were used.

\section{Statistical analysis}

Statistical analysis using Primer (version 5.0) was conducted by an independent physician (GT) who was aware of patient outcome and randomization. The data were expressed as mean $\pm \mathrm{SD}$. The differences in normally distributed values between two groups were assessed using the Student paired $t$ test. Analysis of variance (ANOVA) was used when appropriate. Kaplan-Meier curves were generated to assess the rate of recurrent endometriosis, and the log-rank test was performed to assess the difference in the rate of recurrence between different treatments. The power of study was $80 \%$ and the statistically significant difference was defined as $p<0.05$.

\section{Results}

The mean age of the women taking part in the study was $29 \pm 2.3$ years, and the mean parity was 0.96 . At the time of surgery, we observed a bilateral and a monolateral endometrioma in 37 and $63 \%$ of cases, respectively (Table 1). Also, pelvic adhesions were present in $90 \%$ of cases. No intraoperative complications occurred during surgery; no abdominal conversion was needed.

Before surgery, all women were classified as having stage III or stage IV endometriosis. Endometriosis recurrence occurred in $21(12.5 \%)$ out of the 168 women examined (95\% CI, 8-18\%). One hundred and thirty-one patients reached the 24 month follow-up.

Recurrence of the endometrioma was unilateral and bilateral in 17 and 4 cases, respectively, with the latter observed in Group A and Group B, only.

Eight "non-users" (21\%) did not complete the study: two of them achieved spontaneous pregnancy before 24 months of follow-up, two started to receive OCs therapy because of referred dysmenorrhoeal, three for causes unrelated to the study and one was excluded for cyst persistence at 1 month of follow-up. Twenty-nine patients (22\%) among the user groups (groups B, C and D) did not complete the study: 14 of them for causes unrelated to the study, 11 of them for side effects attributable to OCs therapy, four of them were excluded for cyst persistence at 1 month of follow-up (see also Table 2 and flow diagram).

The main side effects using OCs reported by the 11 women who did not complete the treatment for these reasons, comparable in all three groups of users, exactly, were: headache in ten patients, decreased libido in nine patients, spotting in six patients, water retention in four patients, vaginal dryness in two patients, depression in one patient, acne in one patient, insomnia in one patient. The characteristics of the menstrual bleeding profile during treatment are reported in Table 3 . The rate of endometrioma recurrence was statistically significant in Group A compared with Group B ( $p=0.0021$; relative risk: 4.85; $95 \% \mathrm{CI}$ : 1.77-13.28), Group C ( $p=0.0009$; relative risk: 5.73; $95 \%$ CI: 2.04-16.09) and Group D $(p<0.0001$; relative risk: 9.16; $95 \%$ CI: 3.05-27.53), respectively (Fig. 1). The cumulative probability of cyst recurrence was $74.7 \%$ in group A, $26.5 \%$ in group B, $31.8 \%$ group C and $20.5 \%$

Table 1 Characteristics of the 168 eligible patients

\begin{tabular}{lllll}
\hline & Group A (non-users) & Group B & Group C & Group D \\
\hline Number of patients & 38 & 43 & 44 & 43 \\
Average age \pm SD (years) & $30.2 \pm 2.1$ & $29.6 \pm 2.7$ & $28.5 \pm 1.8$ & $21.4 \pm 2.7$ \\
BMI \pm SD $\left(\mathrm{kg} / \mathrm{m}^{2}\right)$ & $20.8 \pm 2.3$ & $21.2 \pm 2.4$ & & $20.5 \pm 2.5$ \\
American Fertility Society stage & & & 20 & 22 \\
III & 16 & 18 & 24 & 21 \\
IV & 22 & 25 & $4.8 \pm 0.57$ \\
Mean preop. cyst diameter \pm SD $(\mathrm{cm})$ & $4.1 \pm 0.42$ & $4.9 \pm 0.65$ & 17 & $5.1 \pm 0.6$ \\
Bilateral cyst & 13 & 15 & & 17 \\
\hline
\end{tabular}


Table 2 Persistence (at 1 month of follow-up) and recurrence of endometrioma during the 24 months of follow-up

\begin{tabular}{|c|c|c|c|c|c|}
\hline Total number of patients & 1 month & 3 months & 6 months & 12 months & 24 months \\
\hline Group A: 38 pts & $2 / 37$ & $1 / 34$ & $7 / 32$ & $4 / 30$ & $1 / 30$ \\
\hline Group B: 43 pts & $0 / 42$ & $0 / 42$ & $1 / 39$ & $2 / 36$ & $1 / 35$ \\
\hline Group C: 44 pts & $1 / 44$ & $1 / 43$ & $1 / 41$ & $1 / 35$ & $0 / 33$ \\
\hline Group D: 43 pts & $1 / 40$ & $0 / 39$ & $0 / 38$ & $1 / 36$ & $0 / 33$ \\
\hline
\end{tabular}

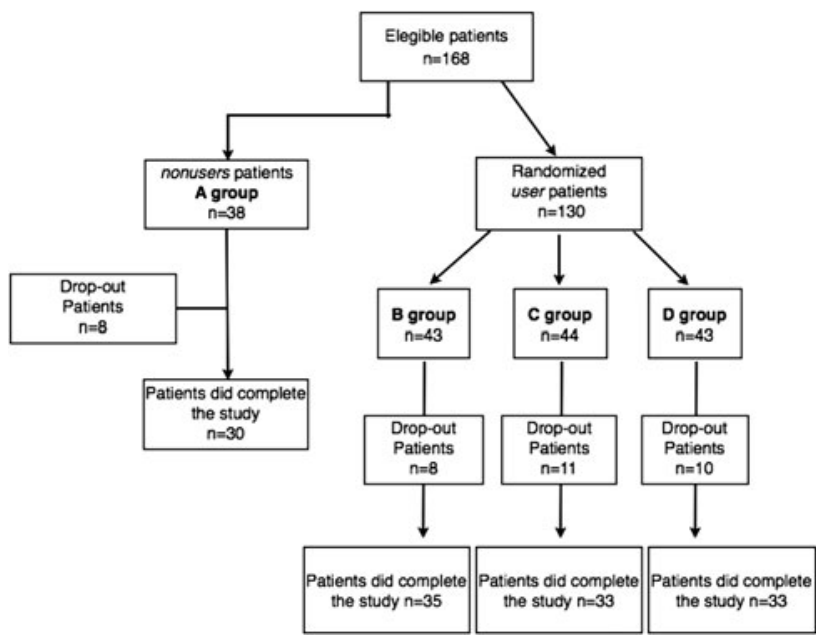

Table 3 Characteristics of the menstrual bleeding profile in the three groups during treatment

\begin{tabular}{llccc}
\hline & Average day flow & Dysmenorrhoea $(\%)$ & Spotting (\%) & Average cadency \\
\hline Group A & 5 & 56 & 7 & 0 \\
Group B & 4 & 7 & 25 & $26 \pm 2$ (OCs users) \\
Group C & 4 & 10 & 32 & $26 \pm 3$ (OCs users) \\
Group D & 3 & 0 & 15 & $27 \pm 2$ (OCs users) \\
\hline
\end{tabular}

in group D. In cases of recurrence, endometrioma diameter was higher in Group A compared with Group B $(3.1 \pm 0.42$ vs $1.9 \pm 0.65 \mathrm{~cm} ; p<0.0001)$, as well as with Group C $(3.1 \pm 0.42$ vs $1.8 \pm 0.57 \mathrm{~cm} ; p<0.0001)$ (Fig. 2).

\section{Discussion}

Effective long-term management of endometriosis is a significant goal, given the debilitating nature of this condition. Recurrence is very common after surgery, varying from 29 to $56 \%$ [2, 10,29], while evidence to support the efficacy and safety of many medications used to treat it is lacking [26].

Retrograde menstruation seems to be the most probable pathogenetic mechanism behind the development of this pathology $[1,5,30,31]$. Decreasing or eliminating the menstrual cycle (and tubaric reflux as a result) through the use of cyclic or continuous OCs may reduce the probability of cyst recurrence or peritoneal reimplantation [5]. The efficacy of postoperative OCs treatment is also supported by the theory that anovulation and moderately low oestrogen concentration may induce atrophy of the endometriotic implants [32], reduce disease-related pain [16], down-regulate cell proliferation and enhance programmed cell death (apoptosis) in the endometrium, which is abnormally low in women with endometriosis [33].

Moreover, it has been hypothesized that since ovulation is causally related to endometriotic cyst development [10, $19,20]$, ovarian suppression may reduce the risk of recurring lesions.

The mechanisms reported above have been the subject of recent studies that assess the effectiveness of long-term 


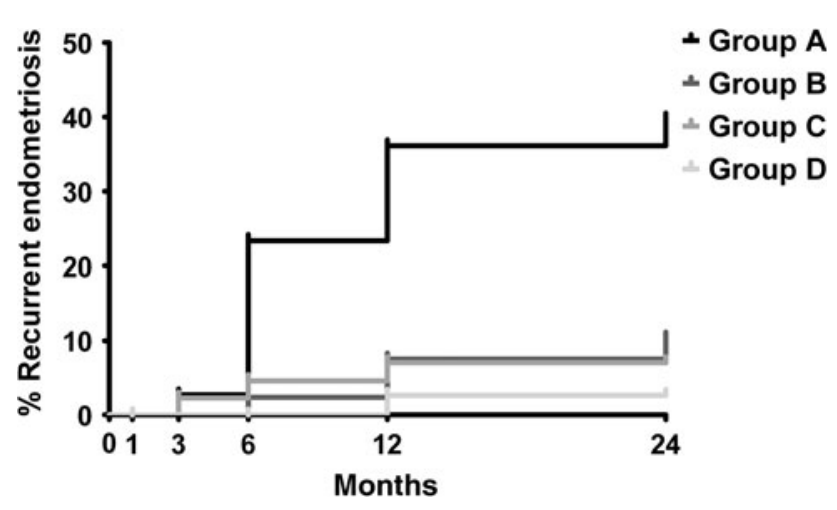

Fig. 1 Kaplan-Meier curves showing the rate of recurrent endometriosis in Group A (nonusers), Group B (monophasic desogestrel), Group C (monophasic gestodene), Group D (Klaira ${ }^{\circledR}$ modified to biphasic)

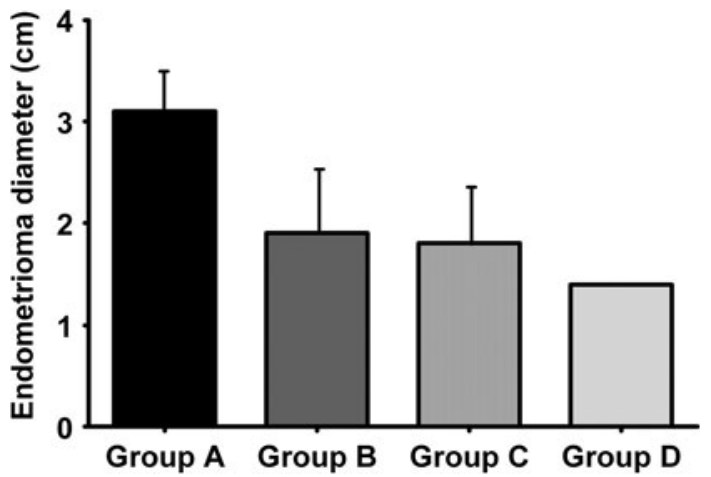

Fig. 2 The bar graph shows the variation in the diameter of recurrent endometrioma (mean $\pm \mathrm{SD}$ ) in the four subgroups who underwent clinical follow-up

OCs administration in preventing ovarian endometrioma recurrence [2], thus offering a valuable alternative to serial surgery [24, 34, 35]. A recent randomized controlled trial [36] examined the effect of postoperative OCs on endometrioma recurrence. The study established that 6-month OCs therapy after surgery tends to prevent recurrence at 12 months after surgery, but does not significantly influence recurrence of the disease at 24- and 36-month followup [36]. In yet another study, it was found that mean postoperative treatment of $\sim 9$ months did not significantly influence recurrence; however, the authors suggested that a longer period of treatment may have an effect on its prevention [6]. Other authors have pointed out that long-term cyclic and continuous OCs use effectively reduces and delays recurrence $[2,10]$. Long-term exposure to OCs has been associated with a greater reduction in the risk of recurrence, which is directly proportional to the duration of therapy [35].

In our sample of patients, postoperative OCs treatment was administered long term. Treatment was performed cyclically because according to several authors [2], no statistically significant difference has been demonstrated between cyclic and continuous use as far as number, size, and growth of recurrent endometrioma are concerned. Furthermore, we chose cyclic administration because it was more easily accepted by patients from the psychological standpoint, since it results in a regular cycle. In literature, a positive trend has also been reported in patients receiving continuous therapy $[2,35]$. This may be due to its constant hormonal environment, which could increase the effectiveness of the therapy [35].

As previously found in other studies [2, 10, 34, 35], we observed a significant reduction in the risk of postoperative endometrioma recurrence in patients who underwent laparoscopic excision and long-term postoperative use of cyclic OCs, compared with patients who did not receive such treatment.

According to another study [24], the progestins contained in the OCs are the most effective hormones against endometriosis, as they may prevent the implantation and growth of regurgitated endometrial cells by inhibiting the expression of matrix metalloproteinases and angiogenesis. This reduces the inflammatory state generated by ectopic endometrium and the consequent immune response [24], which is considered the primary causative factor behind endometriosis [37]. For this reason, we tried to understand whether all progestins play the same role or one type is more active than the others. We decided to compare two monophasic pills containing desogestrel and gestodene, respectively, because they are the only OCs used in European studies dealing with this topic. Moreover, according to some authors [38], the use of desogestrel showed a significant decrease in cell proliferation and an increased apoptosis in the ectopic endometrial tissue. Another study on OCs containing gestodene showed a decrease in aromatase expression in the eutopic endometrium of patients with endometriosis [39]. Aromatase expression in the endometrium seems to play a pivotal role in the development of endometriotic lesions. Because inflammatory mediators such as prostaglandin E2 appear to activate aromatase in the cells of the endometrial stroma, it was hypothesised that the ensuing inflammation caused by the arrival of aromatase-positive cells in the peritoneal cavity would stimulate local oestrogen production, which would in turn facilitate the development of endometriotic lesions by suppressing macrophage phagocytosis [40].

Moreover, our study was also designed to verify the efficacy of an OC containing dienogest, an oral progestin that has recently been investigated for the treatment of endometriosis in a dose-ranging study and in long-term trials in Europe and Japan [26]. Also, it has been approved as a monotherapy for the treatment of this pathology in various countries [26]. Dienogest binds to the progesterone receptor with high specificity and produces a powerful 
progestogenic effect [41]. When administered continuously, it results in anti-ovulatory activity [42], inhibitory effects on cytokine production of endometriotic cells [43], a hypoestrogenic endocrine environment, and decidualization of endometrial tissue and consequent atrophy of the endometriotic lesions [26] without androgenic effects and with minimal changes in lipid and carbohydrate levels [44, 45]. For these reasons, a therapeutic effect against endometriosis was expected.

In a recent multi-centre study, a multiphasic OC with dienogest was considered to be very active in reducing endometriosis pain over the long term; i.e., for at least 24 weeks after discontinuation of treatment [22].

Therefore, we compared two monophasic OCs containing two different progestins and a multiphasic $\mathrm{OC}$ with dienogest. We found that the type of progestin used did not influence recurrence time in treated patients. It is possible that when administered in association with oestrogen, as in the OCs tested in our study, Dienogest is not significantly superior, even though an improved curative trend was observed in the group treated with OCs containing this progestin.

The selection of persistent instead of recurrent cysts was avoided by excluding patients who still presented a cyst at 1-month follow-up. Moreover, scanning was performed only by highly qualified sonographers with extensive experience in the detection of endometriotic lesions.

The protective effect of OCs seems to fade quickly after discontinuation of therapy [10,36], which suggests that the use of OCs be continued until pregnancy is desired [5]. After recurrent surgery, the likelihood of conception has been reported to be almost $50 \%$ lower than after the primary surgery [46-49].

OCs are the only medications which inhibit ovulation, are well tolerated and safe in long-term use, and are also economical [34]. Moreover, OCs prevent the recurrence of pain, improve quality of life, allow conception after discontinuation of treatment, and protect against ovarian cancer associated with endometriosis [50].

Unfortunately, there are some limitations in the current study. One of them is that the number of patients collected is not large enough to draw definitive conclusions. Moreover, we focused the study on cyst recurrence without considering its correlation with symptoms. Further studies on the postoperative use of OCs, considering both aspects, seem therefore necessary to support the suggested therapy.

Another limit of our study is that a good comparison of the effect of the two progestins contained in the OCs with the one with dienogest, to establish the stronger effect on endometrioma recurrence, is not really possible. In fact, the pill with dienogest is combined with natural oestradiol and not with EE, as in the other two OCs, therefore, the nature of the oestrogen could interfere with the different endometriosis inhibition. But, as we did not obtain a significant difference between the three groups, further study on the individual molecules is required in order to really understand the effect of each of them.

Based on the review of medical literature and our observations, a few clinical considerations can be made: (1) in case of recurrence, the endometrioma diameter was higher in patients who had refused postoperative OCs; (2) OCs may potentially reduce the severity of the disease; (3) although no statistical difference was reported for endometrioma diameter among patients using different types of progestins in cyclic OCs therapy, better results were observed in patients treated with dienogest; (4) conservative surgery is the first-line treatment in case of endometrioma, with the second step possibly being to encourage women to become pregnant and/or to prevent endometrioma recurrence with OCs, regardless of the type of progestins used.

Conflict of interest The authors declare to have no financial relationship with any organization or Companies. Authors also declare to have had full control of all primary data and that all coauthors agree to allow the journal to review their data if requested.

\section{References}

1. Eskenazi B, Warner ML (1997) Epidemiology of endometriosis. Obstet Gynecol Clin North Am 24:235-258

2. Seracchioli R, Mabrouk M, Frascà C et al (2010) Long-term cyclic and continuous oral contraceptive therapy and endometrioma recurrence: a randomized controlled trial. Fertil Steril 93:52-56

3. The Practice Committee of the American Society for Reproductive Medicine (2008) Treatment of pelvic pain associated with endometriosis. Fertil Steril 90(Suppl 5):S260-S269

4. Exacoustos C, Zupi E, Amadio A et al (2006) Recurrence of endometriomas after laparoscopic removal: sonographic and clinical follow-up and indication for second surgery. J Minim Invasive Gynecol 13:281-288

5. Vercellini P, Somigliana E, Viganò P, De Matteis S, Barbara G, Fedele L (2010) Post-operative endometriosis recurrence: a plea for prevention based on pathogenetic, epidemiological and clinical evidence. Reprod Biomed Online 21:259-265

6. Koga K, Takemura Y, Osuga Y et al (2006) Recurrence of ovarian endometrioma after laparoscopic excision. Hum Reprod 21:2171-2174

7. Jee BC, Lee JY, Suh CS et al (2009) Impact of GnRH agonist treatment on recurrence of ovarian endometriomas after conservative laparoscopic surgery. Fertil Steril 91:40-45

8. Kikuchi I, Takeuchi H, Kitade M et al (2006) Recurrence rate of endometriomas following a laparoscopic cystectomy. Acta Obstet Gynecol Scand 85:1120-1124

9. Liu X, Yuan L, Shen F et al (2007) Patterns of and risk factors for recurrence in women with ovarian endometriosis. Obstet Gynecol 109:1411-1420

10. Vercellini P, Somigliana E, Daguati R et al (2008) Postoperative oral contraceptive exposure and risk of endometrioma recurrence. Am J Obstet Gynecol 198:504.e1-504.e5

11. ACOG Committee on Practice Bulletins-Gynaecology. ACOG practice bulletin. Medical management of endometriosis. Number 
11, December 1999 (replaces technical bulletin number 184, September 1993) (2000) Clinical management guidelines for obstetrician-gynecologists. Int J Gynaecol Obstet 71(2):183-196

12. Donnez J, Nisolle M, Gillet N et al (1996) Large ovarian endometriomas. Hum Reprod 11:641-646

13. Sutton CJ, Ewen SP, Jacobs SA et al (1997) Laser laparoscopic surgery in the treatment of ovarian endometriomas. J Am Assoc Gynecol Laparosc 4:319-323

14. Yuen PM, Yu KM, Yip SK et al (1997) A randomized prospective study of laparoscopy and laparotomy in the management of benign ovarian masses. Am J Obstet Gynecol 177:109-114

15. Luciano AA, Lowney J, Jacobs SL (1992) Endoscopic treatment of endometriosis-associated infertility. Therapeutic, economic and social benefits. J Reprod Med 37:573-576

16. Chapron C, Vercellini P, Barakat H et al (2002) Management of ovarian endometriomas. Hum Reprod Update 8:591-597

17. Fedele L, Bianchi S, Zanconato G et al (2006) Laparoscopic excision of recurrent endometriomas: long-term outcome and comparison with primary surgery. Fertil Steril 85:694-699

18. Nezhat F, Nezhat C, Allan CJ, Metzger DA, Sears DL (1992) Clinical and histologic classification of endometriomas. Implications for a mechanism of pathogenesis. J Reprod Med 37:771-776

19. Jain S, Dalton ME (1999) Chocolate cyst from ovarian follicles. Fertil Steril 72:852-856

20. Busacca M, Riparini J, Somigliana E et al (2006) Postsurgical ovarian failure after laparoscopic excision of bilateral endometriomas. Am J Obstet Gynecol 195:421-425

21. Somigliana E, Vercellini P, Viganò P et al (2006) Should endometriomas be treated prior to IVF-ICSI cycles? Hum Reprod Update 12:57-64

22. Petraglia F, Hornung D, Seitz C, Faustmann T, Gerlinger C, Luisi S, Lazzeri L, Strowitzki T (2012) Reduced pelvic pain in women with endometriosis: efficacy of long-term dienogest treatment. Arch Gynecol Obstet 285:167-173

23. Seracchioli R, Mabrouk M, Manuzzi L, Vicenzi C, Frascà C, Elmakky A, Venturoli S (2009) Post-operative use of oral contraceptive pills for prevention of anatomical relapse or symptom recurrence after conservative surgery for endometriosis. Human Reprod 24:2729-2735

24. Vercellini P, Fedele L, Pietropaolo G et al (2003) Progestogens for endometriosis: forward to the past. Hum reprod Update 9:387-396

25. Foster RH, Wilde MI (1998) Dienogest. Drugs 56:825-833

26. Schindler AE (2011) Dienogest in long-term treatment of endometriosis. Int J Womens Health 3:175-184

27. The American Fertility Society (1985) Revised American fertility society classification of endometriosis. Fertil Steril 43:351-352

28. Mais V, Guerriero S, Ajossa S, Angiolucci M, Paoletti AM, Melis GB (1993) The efficiency of transvaginal ultrasonography in the diagnosis of endometrioma. Fertil Steril 60:776-780

29. Takamura M, Koga K, Osuga Y et al (2009) Post-operative oral contraceptive use reduces the risk of ovarian endometrioma recurrence after laparoscopic excision. Hum Reprod 24:3042-3048

30. Missmer SA, Cramer DW (2003) The epidemiology of endometriosis. Obstet Gynecol Clin North Am 30:1-19

31. Viganò P, Parazzini F, Somigliana E et al (2004) Endometriosis: epidemiology and aetiological factors. Best Pract Res Clin Obstet Gynecol 18:177-200

32. Rodgers AK, Falcone T (2008) Treatment strategies for endometriosis. Expert Opin Pharmacother 9:243-255

33. Meresman GF, Auge L, Baranao RI et al (2002) Oral contraceptives suppress cell proliferation and enhance apoptosis of eutopic endometrial tissue from patients with endometriosis. Fertil Steril 77:1141-1147
34. Vercellini P, Somigliana E, Vigano P et al (2008) Endometriosis: current and future medical therapies. Best Pract Res Clin Obstet Gynaecol 22:275-306

35. Vercellini P, Frontino G, De Giorgi O et al (2003) Continuous use of an oral contraceptive for endometriosis- associated recurrent dysmenorrhea that does not respond to a cyclic pill regimen. Fertil Steril 80:560-563

36. Muzii L, Marana R, Caruana P et al (2000) Postoperative administration of monophasic combined oral contraceptives after laparoscopic treatment of ovarian endometriomas: a prospective, randomized trial. Am J Obstet Gynecol 183:588-592

37. Vercellini P, Eskenazi B, Consonni D, Somigliana E, Parazzini F, Abbiati A, Fedele L (2011) Oral contraceptives and risk of endometriosis: a systematic review and meta-analysis. Hum Reprod Update 17:159-170

38. Meresman GF, Bilotas M, Abello V, Buquet R, Tesone M, Sueldo C (2005) Effects of aromatase inhibitors on proliferation and apoptosis in eutopic endometrial cell cultures from patients with endometriosis. Fertil Steril 84:459-463

39. Maia H Jr, Casoy J, Correia T, Freitas LA, Pimentel K, Athayde C (2008) The effect of oral contraceptives on aromatase expression in the eutopic endometrium of patients with endometriosis. Gynecol Endocrinol 24:123

40. Maia H Jr, Casoy J, Valente Filho J (2009) Is aromatase expression in the endometrium the cause of endometriosis and related infertility? Gynecol Endocrinol 25:253-257

41. Oettel M, Breitbarth H, Elger W et al (1999) The pharmacological profile of dienogest. Eur J Contracept Reprod Health Care 4:2-13

42. Moore C, Carol W, Graser T, Mellinger U, Walter F (1999) Influence of dienogest on ovulation in young fertile women. Clin Drug Invest 18:271-278

43. Horie S, Harada T, Mitsunari M, Taniguchi F, Iwabe T, Terakawa N (2005) Progesterone and progestational compounds attenuate tumor necrosis factor alpha-induced interleukin-8 production via nuclear factor kappa B inactivation in endometriotic stromal cells. Fertil Steril 83:1530-1535

44. Herkert O, Kuhl H, Sandow J, Busse R, Schini-Kerth VB (2001) Sex steroids used in hormonal treatment increase vascular procoagulant activity by inducing thrombin receptor (PAR-1) expression: role of the glucocorticoid receptor. Circulation 104:2826-2831

45. Köhler G, Faustmann TA, Gerlinger C, Seitz C, Mueck AO (2011) A dose-ranging study to determine the efficacy and safety of 1, 2, and $4 \mathrm{mg}$ of dienogest daily for endometriosis. Erratum in: Int J Gynaecol Obstet 112:257

46. Evers LH, Dunselman GA, Land JA, Bouckaert PX (1998) Endometriosis: prevention of recurrence. In: Kempers RD, Cohen J, Haney AF, Younger JB (eds) Fertility and reproduction medicine, vol 1183. Excerpta medica, International congress series, Amsterdam, pp 387-396

47. Vercellini P, Somigliana E, Daguati R et al (2009) The second time around: reproductive performance after repetitive versus primary surgery for endometriosis. Fertil Steril 92:1253-1255

48. Vercellini P, Somigliana E, Vigano' P et al (2009) Surgery for endometriosis-associated infertility: a pragmatic approach. Hum Reprod 24:254-269

49. Vercellini P, Somigliana E, Vigano' P et al (2009) The effect of second-line surgery on reproductive performance of women with recurrent endometriosis. Acta Obstet Gynecol Scand 88:1074-1082

50. Missmer SA, Hankinson SE, Spiegelman D et al (2004) Reproductive history and endometriosis among premenopausal women. Obstet Gynecol 104:965-974 